\title{
Breast sarcomas. A review of the possible treatment modalities
}

\author{
Nicolae BACALBASA ${ }^{1}$, Olivia IONESCU ${ }^{2}$ \\ 1"Carol Davila" University of Medicine and Pharmacy, Bucharest, Romania \\ 2"Fichtelgebirge" Hospital, Marktredwitz, Bavaria, Germany
}

\begin{abstract}
Background. Breast sarcomas (BS) are rare tumors which origin within the connective tissue of the breast. A clear delimitation from breast carcinoma should be established because the management and evolution and prognosis are different. BS can develop "de novo" - primary BS or can be related to a previously treated malignant breast tumor - for example after breast conservative surgery and adjuvant radiotherapy.

Aim. Owing to the rarity of this tumor type, there is limited evidence with regard to the optimal treatment possibilities. The information comes primary from small retrospective studies so that the treatment principles resemble that of primary mesenchymal malignancies arising in other parts of the body (e.g. leiomyosarcoma, angiosarcoma or liposarcoma). We aimed to make a review of the current treatment possibilities of the BS emphasizing on the type and extent of the surgical treatment, the utility of the axillary lymph node (LN) dissection and the feasibility and benefit of an adjuvant chemotherapy.

Method. We performed an online research on Pubmed using the following key words: "sarcoma", "radiotherapy", "surgery", "chemotherapy". We tried to select the data referring to sarcoma developing with the connective tissue of the breast as well as the information of BS developing after irradiation of the breast in women previously diagnosed with breast cancer $(B C)$ who received adjuvant radiotherapy. We further looked for reports about the role of an adjuvant treatment, namely chemotherapy.

Conclusion. The only treatment proved to have a benefit on the prognosis is the surgical treatment with the achievement of a wide, radical, complete resection (R0-resection). For angiosarcomas, either primary or therapy-related, the margins of resection should be more than $1 \mathrm{~cm}$ for small tumors and more than $3 \mathrm{~cm}$ for larger tumors. Adjuvant chemotherapy has limited benefits and should be applied in selected cases.
\end{abstract}

Abbreviations: $\mathrm{BS}=$ breast sarcoma; $\mathrm{BC}=$ breast cancer; $\mathrm{LN}=$ lymph node; $\mathrm{BCS}=$ breast conservative surgery

Keywords: breast sarcoma, lymph node dissection, chemotherapy

\section{INTRODUCTION AND EPIDEMIOLOGY}

Primary ("de novo") breast sarcomas (BS) are rare tumors with a very low incidence namely 4.6 cases $/ 1,000,000$ women. They represent less than $1 \%$ of all the breast cancer types and less than $5 \%$ of all sarcomas of the soft tissue (1). Treatment-related BS are also rare, data about their incidence fail while the risk of development a sarcoma after BCS and previous breast irradiation is estimated to be between
0.05 and $0.2 \%$ (2). Of all the secondary BS, the most common subtype is the angiosarcoma which most frequently appears in women previously diagnosed with breast cancer. These patients appear to have a cumulative risk of developing an angiosarcoma estimated to be around $59 \%$ (3). Moreover, an adjuvant radiotherapy, either after BCS or mastectomy, increases the risk of developing an angiosarcoma, this risk being directly related to the dosis of radiotherapy 
(4). A prolonged period between the exposure to radiotherapy and the appearance of a BS between 6 and 44 years -, different histopathologic characteristics of the BS than the initial breast tumor as well as the presence of the BS in the field previously irradiated are considered to be mandatory criteria in order to establish the diagnosis of a radiotherapy-induced sarcoma. However, the benefits brought by radiotherapy for $\mathrm{BC}$ patients considerably overcome the risk of a secondary BS $(5,6)$.

When it comes to the histology of the sarcoma, their main histologic characteristic is heterogeneity. As previously stated, owing to the rarity of this disease, the histologic classification is different between experienced pathologist However, the proposed classifications (8) of the BS include the following subtypes: angiosarcoma, fibrosarcoma, undifferentiated pleomorphic sarcoma, myxofibrosarcoma, osteosarcoma and hemangiopericytoma. Of all these, the angiosarcoma has the most aggressive evolution and the poorest prognosis compared to other subtypes (9). One of the factor which influences the prognostic of the BS is the grade of differentiation, the exception being the angiosarcoma whose evolution appears not to be correlated with its grading (10).

Besides the size of the tumor and the stage, the grade of differentiation is one of the factors upon which the treatment is based. The standard treatment of all histological subtypes of sarcomas is surgical, the choice between BCS or mastectomy being influenced by the size of the tumor (11). This review proposes to describe the benefits of the surgical treatment on the prognosis of BS patients as well as to present the current possibilities regarding the adjuvant treatment.

\section{THE SURGICAL TREATMENT}

The surgical treatment is the mainstay in the management of $B S$, hence it should be immediately performed after obtaining the histopathologic confirmation of the diagnosis - through core or punch biopsy - and staging of the disease (12). The choice between BCS or mastectomy should depend on the size of the tumor, patients with BS over $5 \mathrm{~cm}$ in size being most frequently supposed to mastectomy with wide margins of resections (13). If the sarcoma infiltrates one or more ribs, a resection of the chest en bloc with the tumor should be considered. This applies to especially to angiosarcomas which tends to present an infiltrative dissemination affective more than one quadrants of the breast even the chest wall (14). However, the prognosis seems not to be related with the extent of resection but with the size of the excision margins (15).

With regard to the size of the resection margins, the current guidelines recommend "wide excision margins" without specifying the size of the tumor's margins (16). Small angiosarcomas for which a BCS appear to be suitable, the achievement of more than $1 \mathrm{~cm}$ of resection's margins is considered to be adequate. On the contrary, large angiosarcomas, due to their infiltrative pattern, imply wide excisions with margins more than $3 \mathrm{~cm}$ in size (17). In spite of the achievement of wide excision margins and owing to the aggressivity of the sarcomas, the risk of recurrence remains high. A local relapse has been estimated to appear within 1 year after resection. The histopathologic confirmation of an angiosarcoma contraindicates the performance of reconstruction after mastectomy (18). However, a wide excision may imply difficulties in closure of the defect which can be temporary maintained closed (without skin grafts) until the final histopathologic analysis confirms the negative margins (15).

The second most important factor of prognostic - the size of the tumor - has a strong correlation with the prognosis as larger BS, particularly tumors more than $5 \mathrm{~cm}$ in size, are associated with a low overall survival rate (8). On the other hand, the grade of tumor differentiation, has been surprisingly proved to not be related to the outcome although a poor-differentiated tumor generally implies a poorer prognosis (19).

Although the non-epithelial breast malignancy have a lymphatic dissemination, namely in the axillary LNs, BS spread mostly hematogenously or by local invasion (20). The lymphatic spread is rare, excepting angiosarcomas in advanced stages with diffuse metastases. In this situation, the incidence of LN metastases is appreciated to be about $13 \%$ compared with less than $5 \%$ for other subtypes of BS (21). Also in the metastatic situation, an axillary dissection has no impact on the overall and disease-free survival rates (22). Both clinically negative and positive axillas do not require a prophylactic $\mathrm{LN}$-dissection as the result shows in the majority of cases reactive, non-metastatic LNs. The rates of peri- and postoperative complications could be significantly reduced by avoiding to perform an unnecessary axilla dissection. However, if the clinical examinations reveals suspicious axillary 
LNs, the authors recommend the performance of a fine needle biopsy of the LNs with further histopathologic examination for the presence of a carcinosarcoma or a metaplastic carcinoma (23).

BS patients with positive LNs should be offered a radical block axillary dissection if distant metastases (e.g in liver, lungs, other bones) are absent. Axillary LNs metastases from BS are not considered distant metastases and represent a criteria for stage III disease according to the American Joint Committee on Cancer/International Union for Cancer Control (AJCC/UICC) staging system and do not imply the same prognostic as the prognostic of other soft tissue sarcomas with distant metastases (stage IV) (24).

In cases of local relapse after initial mastectomy with complete Ro resection, the surgical treatment appears to have a curative intent especially when it comes to tumors less than $5 \mathrm{~cm}$ in size (7). The surgical resection of the relapse appears to be feasible with favorable two and five years disease specific survival rates. The size of the relapse is the most important predictor of the outcome, tumors more than $5 \mathrm{~cm}$ in size being associated with a high rate of distant metastases and second local recurrences (2). Patients who were initial supposed to BCS and present with a loco-regional recurrence should be counseled about the need of a radical mastectomy. Distant izolated metastases, especially in the lungs, could be approached through metastasectomy. If the functional status, comorbidities, age of the patient contraindicate the performance of a second surgery, the palliative chemotherapy, particularly with taxanes, should be considered $(15,17)$.

\section{THE ROLE OF ADJUVANT AND NEOADJUVANT CHEMOTHERAPY}

Although chemotherapy remains the standard adjuvant treatment in other soft tissue sarcomas, its role and benefits in BS remains unclear. The majority of studies on the role of chemotherapy include patients with soft tissue sarcomas of different histologies so that the data on BS have been extracted from these studies. There are therefore no specific studies addressing only the BS. There is also a disparity in the reported results, some of the studies (20, 25) showing a relative favorable disease-free and overall survival rate or an improved local control of the malignancy, others $(26,27)$ revealing no benefit. For example, two trials studies on the role of adjuvant chemotherapy with doxorubicin and ifosfamid have been by the European Organisation for Research and Treatment of Cancer. Their results did not show a significant advantage on the survival after using this chemotherapeutic agents. On the other hand, one of these two trials have been included in a metaanalysis performed by the Sarcoma Meta-Analysis Collaboration which also aimed at proving the benefits of doxorubicin and ifosfamid. This metaanalysis however resulted in an 11 percent increase of the survival rate for patients who received the respective adjuvant chemotherapy (28).

The decision to apply adjuvant chemotherapy for primary BS should take into consideration the stage, size and location of the sarcoma as well as the patient's functional status, age and comorbidities. Especially, women with tumors more than $5 \mathrm{~cm}$ in diameter and positive LNs should be informed about the possibility to receive chemotherapy. For tumors between 3 and $5 \mathrm{~cm}$ the chemotherapy appear to be beneficial but the decision on chemotherapy in these patients should be discussed in a multidisciplinary tumor conference (29). For treatment-related BS, due to its increased cardiotoxicity, adjuvant chemotherapy with doxorubicine is contraindicated for patients who previously recived anthracycline-based chemotherapy (30). On the other hand if the histology of an angiosarcoma that measures more than $5 \mathrm{~cm}$ in size reveals a possible response to taxanes, a taxane-based adjuvant chemotherapy appears to be feasible (30). For radiotherapy-induced angiosarcomas, an adjuvant chemotherapy can improve local control of the disease especially for patients with high grade tumor, more than $3 \mathrm{~cm}$ in size (31).

Large, poor differentiated sarcomas for which the probability to achieve negative resection margins is low a neoadjuvant chemotherapy could increase the chance to obtain a complete, wide resection. However, this hypothesis applies to non-breast soft tissue sarcomas as the prospective data on the usefulness of a neoadjuvant chemotherapy in BS fail (7). The lack of data could be explained by the generally low response rated to chemotherapy of BS. The decision to apply a neoadjuvant treatment - either radiotherapy or chemotherapy - should also be decided in the multidisciplinary tumor conference (32).

A future direction regarding the non-surgical treatment of large, poor differentiated, 
recurrent sarcomas regards the combination of local hyperthermia and chemotherapy. At present, benefits have been reported after applying hyperthermia and neoadjuvant chemotherapy in patients with soft tissue sarcomas, either arousing in the extremities or in other sites. However, the hyperthermic chemotherapy is currently only experimental (33).

\section{PROGNOSTIC}

As stated above, prognosis of BS is highly influenced by the size of the tumor and the histologic grade. The five-year disease-free survival rate has been estimated to be between $44 \%$ and $66 \%$ while the 5 -year overall survival rate is between $49 \%$ and $67 \%(8,34)$. The reported 10 -years survival rate for grade 1 was $82 \%$, for grade 2 tumors was $62 \%$ while for grade 3 primary BS was $36 \%$. When adjusting for the size of the tumor, tumors less than $5 \mathrm{~cm}$ were associated with a 10 year survival rate of $76 \%$ while tumors between 5 and $10 \mathrm{~cm}$ and larger than $10 \mathrm{~cm}$ with rates of $68 \%$ and $28 \%$ respectively $(7,23)$. The influence of the histologic grade on the prognosis has not been clearly defined. Owing to their aggressivity and rapid evolution (the rapid metastases in the lung, liver, bones, skins and the contralateral breast), all angiosarcomas, especially radiotherapy-induced angiosarcoma, are classified as high-grade tumors with a poor outcome and les treatment possibilities (35, 36). The rates of survival reflect the relative risk of metastases for angiosarcoma and non-angiosarcomas. The reported three-year overall survival rate for primary angiosarcoma was $23 \%$ compared to $60 \%$ for non-angiosarcoma histology. Similarly, the 3 year disease-free survival rate was $7 \%$ compared to $23 \%$ (27).

The most important factor which appears to have a positive impact on the prognostic, especially in the case of radiation-associated BS is the achievement of microscopically negative resection margins (37). Hence, an aggressive surgery aimed at obtaining a complete resection is the most effective method in reducing the rates of mortality and of distant and local recurrences.
A retrospective study in which 23 women with radiation-induced angiosarcoma and 32 women with primary angiosarcoma have been enrolled showed that an $\mathrm{RO}$ resection was associated with 2 - and 5-years survival rates of $64 \%$ and $38 \%$ respectively (38). Other reports showed, however, no influence of the R0-resection on the survival outcome of women with radiotherapy-related breast angiosarcomas with local recurrences and regional or distant metastases appearing 6 months after the complete (39).

As a conclusion, the data on the evolution, treatment and survival rates of breast angiosarcomas, either primary or secondary, remain contradictory and no definite prognosis can be outlined.

\section{CONCLUSIONS}

BS are rare, aggressive tumors, with a rapid evolution and high rated of mortality. The triple conventional therapy - surgery, chemotherapy and radiotherapy - is less effective for this type of breast masses. Of all the three approaches, only the surgical treatment has been proved to exert a positive impact on the survival outcome with the condition of performing a wide, complete excision of the tumor. This is particularly essential for primary and secondary angiosarcomas but the rules applies for all soft tissues sarcomas. Because, the lymphatic dissemination has not been described for BS, the axillary LN dissection is not recommended. The adjuvant treatment consists of chemotherapy with doxorubicin and taxanes and it should be applied for BS more than $5 \mathrm{~cm}$ in size and poor differentiated. It can be offered, with low evidence of benefits, for angiosarcomas between 3 and $5 \mathrm{~cm}$ or in the situation of recurrence after an initial R0-resection. The treatment of the recurrent or metastatic disease should be primary surgical.

Acknowledgement: This work was supported by a grant of the Romanian National Authority for Scientific Research and Innovation, CNCS - UEFISCDI, project number PN-II-RUTE-2014-4-2533.

Conflict of interest: none declared 


\section{REFERENCES}

1. Russell W.O., Cohen J., Enzinger F. et al. A clinical and pathological staging system for soft tissue sarcomas. Cancer 1977; 40:1562.

2. Pandey M., Mathew A., Abraham E.K., Rajan B. Primary sarcoma of the breast. J Surg Oncol 2004; 87:121.

3. Cozen W., Bernstein L., Wang F. et al. The risk of angiosarcoma following primary breast cancer. Br J Cancer 1999; 81:532.

4. Rubino C., Shamsaldin A., Lê M.G. et al. Radiation dose and risk of soft tissue and bone sarcoma after breast cancer treatment. Breast Cancer Res Treat 2005; 89:277.

5. Chahin F., Paramesh A., Dwivedi A. et al. Angiosarcoma of the breast following breast preservation therapy and local radiation therapy for breast cancer. Breast $J$ 2001; 7:120.

6. Mery C.M., George S., Bertagnolli M.M., Raut C.P. Secondary sarcomas after radiotherapy for breast cancer: sustained risk and poor survival. Cancer 2009; 115:4055.

7. Zelek L., Llombart-Cussac A., Terrier P. et al. Prognostic factors in primary breast sarcomas: a series of patients with long-term follow-up. J Clin Oncol 2003; 21:2583.

8. Adem C., Reynolds C., Ingle J.N., Nascimento A.G. Primary breast sarcoma: clinicopathologic series from the Mayo Clinic and review of the literature. $\mathrm{Br} J$ Cancer 2004; 91:237.

9. Yap J., Chuba P.J., Thomas R. et al. Sarcoma as a second malignancy after treatment for breast cancer. Int J Radiat Oncol Biol Phys 2002; 52:1231.

10. Nascimento A.F., Raut C.P., Fletcher C.D. Primary angiosarcoma of the breast: clinicopathologic analysis of 49 cases, suggesting that grade is not prognostic. Am J Surg Pathol 2008; 32:1896.

11. Wiklund T., Huuhtanen R., Blomqvist C. et al. The importance of a multidisciplinary group in the treatment of soft tissue sarcomas. Eur J Cancer 1996; 32A:269.

12. McGowan T.S., Cummings B.J., O'Sullivan B. et al. An analysis of 78 breast sarcoma patients without distant metastases at presentation. Int J Radiat Oncol Biol Phys 2000; 46:383.

13. Shabahang M., Franceschi D., Sundaram M. et al. Surgical management of primary breast sarcoma. Am Surg 2002; 68:673.

14. Monroe A.T., Feigenberg S.J., Mendenhall N.P. Angiosarcoma after breast-conserving therapy. Cancer 2003; 97:1832.

15. Rosen P.P. Sarcoma. In: Rosen's Breast Pathology, Rosen, PP (Eds), Lippincott
Williams and Wilkins, Philadelphia 2001. p.813.

16. Telli M.L., Horst K.C., Guardino A.E., Dirbas F.M., Carlson R.W. Phyllodes tumours of the breast: natural history, diagnosis, and treatment. J Natl Compr Canc Netw. 2007;5:324-330.

17. McGregor G.I., Knowling M.A., Este F.A. Sarcoma and cystosarcoma phyllodes tumors of the breast-a retrospective review of 58 cases. Am J Surg. 1994; 167:477-480.

18. Monroe A.T., Feigenberg S.J., Mendenhall N.P. Angiosarcoma after breast-conserving therapy. Cancer 2003; 97:1832.

19. Moore M.P., Kinne D.W. Breast sarcoma. Surg Clin North Am. 1996; 76:383-392.

20. Fields R.C., Aft R.L., Gillanders W.E. et al. Treatment and outcomes of patients with primary breast sarcoma. Am J Surg 2008; 196:559.

21. Kaklamanos I.G., Birbas K., Syrigos K.N. et al. Breast angiosarcoma that is not related to radiation exposure: a comprehensive review of the literature. Surg Today 2011; 41:163.

22. Nascimento A.F., Raut C.P., Fletcher C.D. Primary angiosarcoma of the breast: clinicopathologic analysis of 49 cases, suggesting that grade is not prognostic. Am J Surg Pathol 2008; 32:1896.

23. Gutman H., Pollock R.E., Ross M.I. et al. Sarcoma of the breast: implications for extent of therapy. The M. D. Anderson experience. Surgery 1994; 116:505.

24. http://www.uicc.org/sites/main/files/private/ TNM_Classification_of_Malignant Tumours_Website_15\%20MAy2011.pdf

25. Bousquet G., Confavreux C., Magné N. et al. Outcome and prognostic factors in breast sarcoma: a multicenter study from the rare cancer network. Radiother Oncol 2007; 85:355.

26. Lagrange J.L., Ramaioli A., Chateau M.C. et al. Sarcoma after radiation therapy: retrospective multiinstitutional study of 80 histologically confirmed cases. Radiation Therapist and Pathologist Groups of the Fédération Nationale des Centres de Lutte Contre le Cancer. Radiology 2000; 216:197.

27. Confavreux C., Lurkin A., Mitton N. et al. Sarcomas and malignant phyllodes tumours of the breast - a retrospective study. Eur $\mathrm{J}$ Cancer 2006; 42:2715.

28. Pervaiz N., Colterjohn N., Farrokhyar F. et al. A systematic meta-analysis of randomized controlled trials of adjuvant chemotherapy for localized resectable soft-tissue sarcoma. Cancer 2008; 113:573.

29. Palta M., Morris C.G., Grobmyer S.R. et al. Angiosarcoma after breast-conserving therapy: long-term outcomes with hyperfractionated radiotherapy. Cancer 2010; 116:1872.

30. Depla A.L., Scharloo-Karels C.H., de Jong M.A. et al. Treatment and prognostic factors of radiation-associated angiosarcoma (RAAS) after primary breast cancer: a systematic review. Eur J Cancer 2014; 50:1779.

31. Casali P.G., Blay J.Y., ESMO/ CONTICANET/EUROBONET Consensus Panel of experts. Soft tissue sarcomas: ESMO Clinical Practice Guidelines for diagnosis, treatment and follow-up. Ann Oncol 2010; 21 Suppl 5:v198.

32. Trent II J.C. $2^{\text {nd }}$, Benjamin R.S., Valero V. Primary soft tissue sarcoma of the breast. Curr Treat Options Oncol 2001; 2:169.

33. Thalhammer M. et al. Hyperthermia as a therapeutic option in recurrent breast sarcoma. Eur J Surg Oncol 2006; 32(S1): S64. Data presented at the $13^{\text {th }}$ Congress of the European Society of Surgical oncology, Venice Italy, November 30 December 2, 2006. Abstract available online at http://www.sciencedirect.com. ezp-prod1.hul.harvard.edu/science? $\mathrm{ob}=$ Mlmg\&_imagekey=B6WF44MM7RM7-7N-1\&_cdi $=6784 \&$ user=209690\&_pii=S0748798306706503\& _origin=browse\&_zone=rsit_list_item \& _coverDate $=11 \% 2 \mathrm{~F} 30 \% 2 \mathrm{~F} 2006$ \& sk=999679999.8998\&wchp= dGLbVzW-zSkzS\&md5= 9532dfbb86f060bb0bc3c495f9aceef2\&ie=/ sdarticle.pd. (Accessed on January 25, 2011).

34. Ciatto S., Bonardi R., Cataliotti L., Cardona G. Sarcomas of the breast: a multicenter series of 70 cases. Neoplasma 1992; 39:375.

35. Luini A., Gatti G., Diaz J. et al. Angiosarcoma of the breast: the experience of the European Institute of Oncology and a review of the literature. Breast Cancer Res Treat 2007; 105:81.

36. Shet T., Malaviya A., Nadkarni M. et al. Primary angiosarcoma of the breast: observations in Asian Indian women. J Surg Oncol 2006; 94:368.

37. Donnell R.M., Rosen P.P., Lieberman P.H. et al. Angiosarcoma and other vascular tumors of the breast. Am J Surg Pathol $1981 ; 5: 629$.

38. Vorburger S.A., Xing Y., Hunt K.K. et al. Angiosarcoma of the breast. Cancer 2005; 104: 2682

39. Seinen J.M., Styring E., Verstappen V. et al. Radiation-associated angiosarcoma after breast cancer: high recurrence rate and poor survival despite surgical treatment with R0 resection. Ann Surg Oncol 2012; 19:2700. 